\title{
SPONDYLOARTHROPATHIES
}

\section{Gut dysbiosis in ankylosing spondylitis}

E:

vidence is accumulating for

a role of intestinal dysbiosis

in the pathogenesis of several

spondyloarthropathies, including psoriatic arthritis (PsA) and ankylosing spondylitis

(AS). Newly published evidence now reveals a discrete microbial signature in the terminal ileum of patients with AS, versus healthy controls. "This finding is consistent with AS being driven by bacterial interactions with the immune system in the gut mucosa, and our study is the first time that this has been tested by sequencing microbial profiling methods," comments Matt Brown of the University of Queensland Diamantina Institute, corresponding author of the study.

The researchers obtained terminal ileum biopsy samples from nine healthy controls and nine TNF-inhibitor-naive patients with AS and disease duration $\leq 48$ months. "The main reason to focus on the ileum is that most of the inflammation is localized there," comments Dirk Elewaut of Ghent University Hospital, Belgium, who was not involved in the study. As Brown confirms, "70\% of patients with AS have low-grade inflammation in the terminal ileum, which is related to the clinical course."

Microbial populations were characterized by high-throughput barcoded amplicon sequencing of microbial 16S ribosomal RNA genes, a culture-independent analysis technique. Samples from patients with AS showed more diverse microbial communities than healthy controls, and the actual genera present also differed substantially. The AS group had a markedly increased abundance of Lachnospiraceae, Veillonellaceae, Prevotellaceae,

Porphyromonadaceae and Bacteroidaceae, and a markedly decreased abundance of Ruminoccoccaceae and Rikenellaceae. Unlike in Crohn disease, microbial biomass did not differ between patients with AS and controls, and rigorous statistical analyses confirmed that disease status was the factor most strongly linked to these differences.
Microbial interactions also seem to shape the AS microbial community - the researchers describe positive correlations between Lachnospiraceae and Ruminoccoccaceae, and negative correlations between Veillonellaceae and Prevotellaceae. Interestingly, Klebsiella species were not linked with AS in this study, unlike in previous reports.

"Although the number of patients is small, it appears that a microbial signature for these patients is present and characterized by microorganisms previously implicated in other rheumatic and inflammatory diseases-Prevotella has been found in both Crohn disease and rheumatoid arthritis, and Ruminoccocus species are decreased in inflammatory bowel disease and PsA," notes Jose Scher of the Microbiome Center for Rheumatology and Autoimmunity, NY University School of Medicine, an independent commentator.

Costello et al. identified a core population of 100 microbial species present in all nine patients with AS, most of which (with the exception of Clostridia) are also AS indicator species. These observations suggest that the core microbiome drives the AS microbial signature. The range of organisms included indicate a potential pathogenetic role for bacterial invasion of epithelial cells, antimicrobial (butirosin, neomycin) biosynthesis pathways, and disordered secondary bile acid synthesis pathways.

Increases in Prevotellaceae and decreases in Rikenellaceae also occur in the HLA-B27 transgenic rat model of spondyloarthritis. "Seminal work showed that these animals develop colitis and spondylitis only when raised in conventional cages," points out Scher. "Rendering the rats germ-free abrogates disease, and the phenotype can be restored by introducing gut bacteria, although the presence of HLA-B27 alone might drive some of the gut microbial composition in this model," he continues. Accordingly, given the strong association of HLA-B27 with AS,

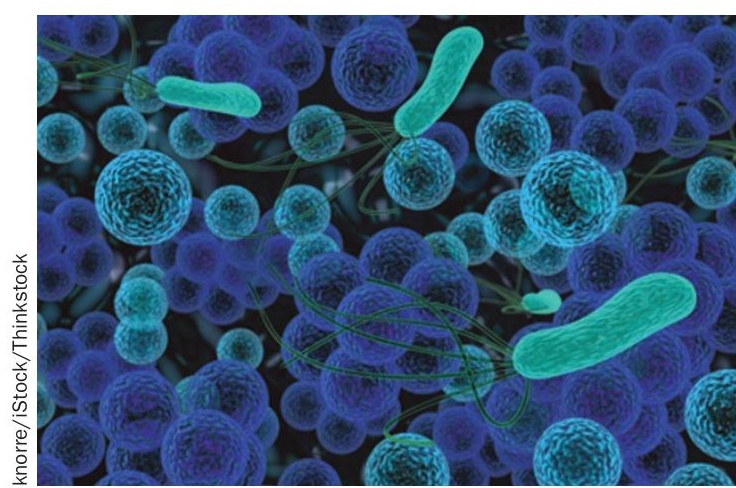

some researchers have hypothesized that HLA-B27 promotes AS via its effects on the gut microbiome, which in turn drives arthritogenic immune mechanisms such as IL-23 production. "It is possible that the changes we observed are due to effects of AS itself on the gut microbial profile, or that immunogenetic associations of AS affect the microbial profile in a manner independent of the mechanisms by which it causes AS," comments Brown, noting that the complex interplay between the host genome, host immune system and gut microbiome remains to be unravelled.

"We are expanding the sample size to enable us to better define the relationship between the microbiome and disease activity, genetic factors associated with AS, mucosal immunological parameters," states Brown, "and to identify more precisely which bacteria or what bacterial profile causes AS." Finally, Scher notes, "Studying the gut microbiome in patients with new-onset AS, compared to HLA$\mathrm{B} 27^{+}$healthy controls, will be required, as well as validating these results in geographically diverse populations."

\section{Caroline Barranco}

Original article Costello, M.-E. et al. Intestinal dysbiosis in
ankylosing spondylitis. Arthritis Rheumatol. doi:10.1002/
art.38967
Further reading Scher, J. U. et al. Decreased bacterial
diversity characterizes an altered gut microbiota in psoriatic
arthritis and resembles dysbiosis of inflammatory bowel
disease. Arthritis Rheumatol. doi:10.1002/art.38892

